\title{
CINESIOTERAPIA PREVINE OMBRO DOLOROSO EM PACIENTES HEMIPLÉGICOS/PARÉTICOS NA FASE SUB- AGUDA DO ACIDENTE VASCULAR ENCEFÁLICO
}

\author{
Agnes Irna Horn ${ }^{1 a}$, Sissy Veloso Fontes ${ }^{1 a}$, Sebastião Marcos Ribeiro de Carvalho ${ }^{2 a b}$, \\ Rubens Augusto Brazil Silvado ${ }^{4 c}$, Pedro Marco Karan Barbosa ${ }^{3 c}$, Alcides Durigan Jrc, \\ Álvaro Nagib Atallah ${ }^{4 a}$, Marcia Maiumi Fukujima ${ }^{4 a}$, Gilmar Fernandes do Prado ${ }^{4 a}$
}

\begin{abstract}
RESUMO - Contexto: Ombro doloroso é freqüente em pacientes com hemiplegia/hemiparesia (H/P) por acidente vascular encefálico (AVE), dificultando a recuperação neuromotora gerando incapacidade funcional. Objetivo: Estudar tratamento fisioterapêutico para a prevenção da dor no ombro com H/P em pacientes com AVE na fase sub-aguda, e analisar desfechos secundários (força muscular do ombro acometido e movimentos funcionais ativos básicos). Método: Estudaram-se 21 pacientes (12 homens, 9 mulheres; idades 26 a 87 anos) com H/P. 0 tratamento fisioterapêutico consistiu de 30 minutos diários de cinesioterapia, desde as 48 horas após o AVE até a alta hospitalar. Os pacientes foram avaliados antes e após o tratamento em relação à presença ou ausência de dor no ombro H/P, à força dos diversos grupos musculares do ombro e quanto aos movimentos funcionais de transferência e manutenção postural básica. Resultados: Nenhum paciente apresentava dor no ombro H/P na alta $(p<0,001)$. A força muscular aumentou significativamente em relação à elevação, protusão, abdução e flexão do ombro $(p<0,001)$. Houve melhora dos movimentos funcionais: decúbito dorsal para lateral, decúbito lateral para sentado e manter-se sentado $(p<0,001)$. Conclusão: A cinesioterapia na fase aguda do AVE preveniu a dor no ombro H/P e favoreceu a recuperação motora.
\end{abstract}

PALAVRAS-CHAVE: acidente vascular cerebral, fisioterapia, ombro doloroso.

Kinesiotherapy prevents shoulder pain in hemiplegic/paretic patients on sub-acute stage post-stroke

ABSTRACT - Background: Painful shoulder is considered the most common complication of hemiplegic/parethic $(\mathrm{H} / \mathrm{P})$ stroke patients. It is a negative factor for neuromotor recovery. Purpose: To study the effects of kinesiotherapy treatment on shoulder pain prevention, and as a secondary endpoints, to analyze muscle strength of $\mathrm{H} / \mathrm{P}$ shoulder looking at basic functional active mobility. Method: Twenty one inpatients (12 men, 9 women) aged 26 to 87 years, with post-stroke H/P were submitted to thirty minutes daily program kinesiotherapy, started at 48 hours post-stroke up to their hospital discharge. Patients were evaluated pre and post treatment according to the presence or absence of shoulder pain, movements and shoulder strength, and for presence or absence of basic functional movements. Results: No patient complaining of shoulder pain at the hospital discharge $(p<0.001)$. The muscle strength improved signifcantly for elevation, protusion, abduction and flexion of the shoulder $(p<0.001)$. There was improvement also for functional mobility on moving from dorsal to lateral recumb, from lateral recumb to a seated position and in keeping the seated position $(p<0.001)$. Conclusion: Kinesiotherapy in acute phase of stroke prevented shoulder pain.

KEY WORDS: stroke, physiotherapy, shoulder pain.

A recuperação de um paciente com hemiplegia/ hemiparesia $(H / P)$, considerada a principal causa de incapacidade grave na sociedade atual, constitui-se em um grande desafio, tanto pela complexidade das funções perdidas, quanto pela alta incidência de dor no ombro $\mathrm{H} / \mathrm{P}^{1-3}$, resultando em sofrimento e impacto negativo na sua recuperação global ${ }^{4-7}$. Cerca de três quartos dos pacientes sofrem de dor no ombro durante o primeiro ano após o acidente vascular encefálico (AVE) e, em aproximadamente $20 \%$ deles, a dor pode surgir na $1^{\text {a }}$ e $2^{\text {a }}$ semana pós ictal, prolongando o tempo de internação $0^{8-10}$.

\footnotetext{
aUniversidade Federal de São Paulo (UNIFESP) São Paulo SP, Brasil, bUniversidade de Marília (UNIMAR) Marília SP, Brasil, cFaculdade de Medicina de Marília (FAMEMA), Marília SP, Brasil: ${ }^{1}$ Fisioterapeuta; ${ }^{2}$ Estaticista; ${ }^{3}$ Enfermeiro; ${ }^{4}$ Médico.
}

Recebido 2 Janeiro 2003, recebido na forma final 21 Março 2003. Aceito 9 Abril 2003.

Fta. Agnes Irna Horn - Disciplina de Neurologia, UNIFESP - Rua Botucatu 740 - 04023-900 São Paulo SP - Brasil. E-mail: hornagnes@hotmail.com 
O quadro caracteriza-se por dor no ombro e perda progressiva da amplitude de movimento articu$\operatorname{lar}(\mathrm{ADM})^{4,11}$, possivelmente devido aos mecanismos de desalinhamento do ombro, movimentação incorreta, imobilidade, manuseio e posicionamento inadequado do braço acometido 2,11-14. Apesar das causas, fatores de risco e tratamento serem desconhecidos, há consenso quanto a importância e a necessidade de profilaxia ${ }^{1,15,16}$. Tanto a eletro-estimulação, quanto o uso de tipóias mostraram resultados pouco favoráveis ${ }^{17-19}$. A cinesioterapia é o procedimento mais utilizado; no entanto, nem todos os exercícios são benéficos ${ }^{1,2,20-22}$. Comparando-se três tipos de exercícios passivos (polias e roldanas, skate e exercícios realizados pelo terapeuta), observou-se que os pacientes submetidos a exercícios realizados pelo fisioterapeuta apresentaram uma incidência menor de dor no ombro $\mathrm{H} / \mathrm{P}^{21}$.

O objetivo deste estudo foi avaliar os efeitos do tratamento fisioterapêutico com cinesioterapia em pacientes H/P por AVE na fase sub-aguda em relação a: dor e força muscular no ombro acometido, presença ou ausência de movimentos funcionais ativos básicos antes e após o tratamento.

\section{MÉTODO}

Pacientes. Vinte e um pacientes com $\mathrm{H} / \mathrm{P}$, internados nas enfermarias dos Hospitais de Clínicas, Santa Casa de Misericórdia e São Francisco de Marília, foram submetidos a programa diário de 30 minutos de tratamento fisioterapêutico com cinesioterapia iniciado nas primeiras 48 horas após a ocorrência do AVE até o momento da alta hospitalar. Foram incluídos pacientes com diagnóstico clínico de primeiro episódio de AVE com H/P e em condições clínicas de realização do tratamento ativo com cinesioterapia. Foram excluídos os pacientes com história anterior de dor ou trauma no ombro acometido, dificuldade de comunicação grave e que não receberam pelo menos 5 dias consecutivos de tratamento.

Instrumentos de avaliação: Avaliaram-se no ombro com H/P a presença ou ausência de dor, os graus de força muscular dos movimentos de elevação, protusão, flexão e abdução do ombro (zero a 5) ${ }^{23}$, e a capacidade de realizar ativamente os movimentos funcionais básicos (mudança de decúbito dorsal para lateral, da posição deitado para sentado e a manutenção da postura sentada) ${ }^{24}$. Para as variáveis passar para o decúbito lateral, passar para sentado e manter-se sentado adotou-se a seguinte escala de escores: (0) Não realiza o movimento/não mantém a posição; (1) Realiza o movimento com dificuldade/mantém a posição com dificuldade; (2) Realiza o movimento/ mantém-se na posição. Perguntou-se ao paciente se havia dor durante o repouso e durante a movimentação passiva do ombro (flexão-extensão, abdução-adução e rotação medial e lateral). Antes da alta hospitalar, todos os pacientes foram novamente avaliados de acordo com os mesmos critérios estabelecidos para a avaliação inicial.

Protocolo de tratamento: O tratamento fisioterapêutico consistiu em cinesioterapia e orientações segundo as diretrizes básicas do método neuro-evolutivo (NDT) do conceito Bobath ${ }^{11,14}$. Os principais movimentos cinesioterapêuticos utilizados no programa de tratamento aplicado foram os seguintes Paciente deitado: 1) Mobilização da escápula; 2) Protusão do ombro com flexão anterior e rotação do tronco superior; 3) Flexão do braço; 4) Movimento seletivo do braço; 5) Extensão de quadril, descarga e transferência de peso; 6) Passar para o decúbito lateral; 7) Depressão de ombro com flexão lateral de tronco; 8) Passar para sentado. Paciente sentado: 9) Flexão e extensão com rotação de tronco associado a movimentos do membro superior; 10) Movimentação auto-assistida. $O$ paciente foi orientado a posicionar o ombro acometido em leve protusão e em alinhamento entre o úmero e a escápula e em simetria com o ombro contralateral, evitar tracionar o braço nas transferências e atentar ao acompanhamento da escápula durante a movimentação do membro superior.

Método estatístico: Os resultados foram analisados utilizando o teste de Wilcoxon para amostras pareadas, comparando-se os indivíduos com AVE, antes e após o tratamento fisioterapêutico, para todas as variáveis do estudo, exceto a dor, que foi analisada segundo sua probalidade esperada (teste binomial). Para a rejeição da hipótese de nulidade, adotou-se $\alpha<5 \%{ }^{25}$.

Consentimento informado: $\mathrm{O}$ estudo foi aprovado pelo Comitê de Ética das instituições envolvidas e todos os pacientes assinaram o termo de consentimento informado.

\section{RESULTADO}

Vinte e um pacientes fizeram parte desse estudo (12 homens e 9 mulheres). A idade variou de 26 a 87 anos, com média de 52,95 anos, mediana 69 e moda 68 e 80 anos. Todos os pacientes receberam tratamento fisioterapêutico diário de 30 minutos durante o tempo de internação hospitalar, que variou entre 5 e 23 dias. Antes de iniciar o tratamento fisioterapêutico, todos os pacientes apresentavam déficit motor no membro superior e nenhum deles apresentava dor no ombro do hemicorpo acometido pelo AVE.

Os 21 pacientes (100\%) evoluíram sem apresentar dor no ombro $\mathrm{H} / \mathrm{P}$ até o momento da alta hospitalar $(p<0,001)$.

Após o tratamento fisioterapêutico houve melhora da força muscular dos movimentos de elevação $(p<0,001)$, protusão $(p<0,001)$, flexão $(p<0,001)$ e abdução $(p<0,001)$ do ombro acometido.

Também houve melhora dos movimentos funcionais de passar dos decúbitos dorsal para o lateral $(p<0,001)$, do lateral para sentado $(p<0,001)$ e manter-se sentado $(p<0,001)$ ao final do tratamento. 


\section{DISCUSSÃO}

O presente estudo adveio da necessidade de estabelecer medidas eficazes na prevenção da dor no ombro H/P. É uma complicação muito freqüente e suas causas e tratamento adequado ainda não estão claramente estabelecidos ${ }^{26}$. Além disso, resulta em grande impacto negativo sobre a recuperação funcional de pacientes que sobrevivem a um $\mathrm{AVE}^{4-7}$. Devido à importância e à necessidade de medidas profiláticas precoces, apontadas por vários autores ${ }^{1,15,16}$, o tratamento fisioterapêutico proposto para esse estudo foi iniciado nas primeiras 48 horas pois, em cerca de $20 \%$ dos pacientes, a dor no ombro H/ $\mathrm{P}$ pode surgir já na $1^{\text {a }}$ e $2^{\text {a }}$ semana pós ictal ${ }^{8-10}$.

Grande parte dos estudos encontrados sobre o assunto não pode ser utilizada como parâmetros de comparação, devido a numerosas variações como falta de referência em relação à prevenção da dor e movimentos que envolvem o ombro, diferentes critérios de inclusão e falta de descrição detalhada do tratamento ${ }^{13,27}$. Uma revisão sistemática sobre o uso da eletro-estimulação no ombro doloroso H/P mostrou que esse tratamento exerceu efeito positivo sobre a força e o tônus muscular mas, os resultados não mostraram evidência de que a eletro-estimulação possa reduzir a dor nesses pacientes ${ }^{17}$. Outra revisão sistemática sobre a eficácia dos métodos de tratamento do ombro doloroso $\mathrm{H} / \mathrm{P}$ disponíveis mostrou que o tratamento adequado continua desconhecido ${ }^{26}$.

Nossos resultados favoráveis mostraram que o tratamento fisioterapêutico utilizado contribuiu na prevenção da dor no ombro H/P durante o período de internação até o momento da alta hospitalar, diferentemente de outros estudos que obtiveram resultados menos favoráveis na prevenção do ombro doloroso na $\mathrm{H} / \mathrm{P}^{18-20}$. Os resultados positivos do tratamento utilizado na recuperação motora sugerem que ele exerceu efeito positivo na função neuromotora do paciente.

Apesar de haver controvérsia sobre a relação entre a falta de força muscular e a ocorrência de dor no ombro $\mathrm{H} / \mathrm{P}^{3,8}$, observou-se, neste estudo, que a ausência ou a falta de força muscular isolada não foi um fator preditivo de dor, pois 4 dos 21 pacientes mantiveram o quadro de ausência total dos movimentos no lado acometido, e um paciente manteve a mesma dificuldade inicial nos movimentos funcionais.

Os resultados do nosso estudo parecem estar relacionados com outros aspectos que são considerados importantes, como o início precoce do tratamento, o posicionamento e o manuseio correto do paciente, principalmente nas transferências posturais, conforme descritos por outros autores ${ }^{11-}$ 15. Com a musculatura flácida, decorrente da perda do controle motor na fase inicial do AVE, o ombro torna-se muito suscetível a graus variados de lesões, principalmente ao estiramento e ao posicionamento inadequado do membro superior. Observou-se, no entanto, que apesar de todos os pacientes terem evoluído sem dor no ombro H/P, em alguns deles houve dificuldade em manter o posicionamento orientado pelo terapeuta, fato já observado anteriormente por alguns autores ${ }^{10}$.

Com a realização criteriosa dos movimentos cinesioterapêuticos dentro dos princípios biomecânicos normais, buscou-se evitar os mecanismos de fricção, tração e pinçamento, considerados fatores mecânicos de lesões no ombro H/P $\mathrm{P}^{11-14}$, pois está estabelecido que, nem todos os exercícios físicos são benéficos para este problema ${ }^{1,2,20-22}$. Outro aspecto que favoreceu os resultados foi a manutenção da ADM do ombro $\mathrm{H} / \mathrm{P}$ livre para os movimentos, visto que estudos mostram que o ombro doloroso, geralmente vem acompanhado com a perda progressiva de sua amplitude de movimento articular ${ }^{3,8-10}$.

Embora baseado numa série de casos, esse estudo inicial permitiu concluir que o programa de tratamento fisioterapêutico elaborado (cinesioterapia na fase aguda do AVE) preveniu a dor no ombro H/ $P$ na fase hospitalar, e, aparentemente favoreceu a recuperação motora dos pacientes, sendo recomendável a realização de ensaio clínico randomizado com avaliação cega dos desfechos por pelo menos dois observadores e com seguimento mais prolongado para avaliar o efeito deste tratamento a médio e longo prazo.

\section{REFERÊNCIAS}

1. Griffin J, Reddin G. Shoulder pain in patients with hemiplegia. Phys Ther 1981;61:1041-1045.

2. Cailliet R. O ombro na hemiplegia. São Paulo: Manole, 1981:1-128.

3. Bohannon RW, Larkin PA, Smith MB, Horton MG. Shoulder pain in hemiplegia: statistical relationship with five variables. Arch Phys Med Rehabil 1986;67:514-516.

4. Braun RM, West F, Mooney V, et al. Surgical treatment of the painful shoulder contrature in the stroke patient. J Bone Joint Surg Am 1971,53:1307-1312.

5. Gowland C. Recovery of motor function following stroke: profile and predictors. Physiother Can 1982;34:77-84.

6. De Courval LP, Barsauskas A, Berenbaum B, et al. Painful shoulder in the hemiplegic and unilateral neglect. Arch Phys Med Rehabil 1990;71:673-676.

7. Wyller TB, Sveen U, Sodring KM, Pettersen AM, Bautz-Holter E. Subjective well-being one year after stroke. Clin Rehabil 1997;11:139-145.

8. Roy CW, Sands M, Hill L. Shoulder pain in acutely admitted hemiplegics. Clin Rehabil 1994;8:334-340.

9. Van Ouwernaller C, Laplace PM, Chantraine A. Painful shoulder in hemiplegia. Arch Phys Med Rehabil 1986;67:23-26. 
10. Wanklyn P, Forster A, Young J. Hemiplegic shoulder pain (HSP): natural history and investigation of associated features. Disabil Rehabil 1996;18:497-501.

11. Davies PM. Hemiplegie: Anleitung zu einer umfassenden Behandlung von Patienten mit Hemiplegie. Berlin: Springer-Verlag, 1996:228-270.

12. Ryerson S, Levit K. The shoulder in hemiplegia. In Donatelli RA, (ED). Physical therapy of the shoulder. 3.Ed. Philadelphia: Churchill Livingstone, 1997:205-226.

13. Carr JH, Shepherd RB. Programa de reaprendizagem motora para o hemiplégico adulto. São Paulo: Manole, 1988:35-62.

14. Bobath B. Die Hemiplegie Erwachsener: Befundaufnahme, Beurteilung und Behandlung. Stuttgart: Georg Thieme Verlag, 1993: 2-224.

15. Moskowitz H, Goodman CR, Smith E, Balthazar E, Mellins, HZ. Hemiplegic shoulder. N Y State J Med 1969;69:548-550.

16. Snels IAK, Beckerman H, Lankhorst GJ. Treatment of hemiplegic shoulder pain in the Netherlands: results of a national survey. Clin Rehabil 2000;14:20-27.

17. Price CIM, Pandyan AD. Electrical stimulation for preventing and treating post-stroke shoulder pain (Cochrane Review). In: The Cochrane Library, Issue 2, 2001. Oxford: Update Software.
18. Hurd MM, Farrels HK, Waylonis GW. Shoulder sling for hemiplegia: friend or foe? Arch Phys Med Rehabil 1974;55:519-522.

19. Linn SL, Granat MH, Lees KR. Prevention of shoulder subluxation after stroke with eletrical stimulation. Stroke 1999;30:963-968.

20. Najenson T, Yacubovich E, Pikielni SS. Rotator cuff in shoulder joints of hemiplegic patients. Scand J Rehabil Med 1971;3:133-137.

21. Kumar R, Metter EJ, Mehta AJ, Chew T. Shoulder pain in hemiplegia. Am J Phys Med Rehabil 1990;69:205-208.

22. Brocklehurst JC, Andrews K, Richards B, Laycock, PJ. How much physical therapy for patients with stroke? Br Med J 1978;1:1307-1310.

23. Daniels L, Worthington. Provas de Função muscular: técnicas de exame manual. Rio de Janeiro: Guanabara; 1987.

24. Collin C, Wade D. Assessing motor impairment after stroke: a pilot reliability study. J Neurol Neurosurg Psychiatry 1990;53:576-579.

25. Armitage P, Berry G. Estatística para la investigación biomédica. 3.Ed. Madrid: Harcourt Brace, 1997.

26. Snels IAK, Dekker JHM, van der Lee JH, Langhorst GJ, Beckerman H, Bouter LM. Treating patients with hemiplegic shoulder pain. Am J Phys Med Rehabil 2002;81:150-160.

27. Ashburn A. Physiotherapy in the rehabilitation of stroke: a review. Clin Rehabil 1993;7:337-345. 